\title{
Common fixed point theorems of integral type contraction on metric spaces and its applications to system of functional equations
}

Muhammad Sarwar ${ }^{1}$, Mian Bahadur Zada ${ }^{1 *}$ and Inci M Erhan²

"Correspondence:

mbz.math@gmail.com

${ }^{1}$ Department of Mathematics,

University of Malakand, Chakdara Dir(L), Pakistan

Full list of author information is available at the end of the article

\begin{abstract}
In this article, using the common (CLR) property, common fixed point results for two pairs of weakly compatible mappings satisfying contractive condition of integral type on metric spaces are established. Furthermore, the existence and uniqueness of common solution for system of functional equations arising in dynamic programming are discussed as an application of a common fixed point theorem presented in this paper.
\end{abstract}

MSC: $47 \mathrm{H} 10 ; 54 \mathrm{H} 25$

Keywords: contractive mappings of integral type; weakly compatible mappings; common fixed point; system of functional equations; dynamic programming; common (E.A) property; common (CLR) property

\section{Introduction and preliminaries}

Throughout this paper, we assume that $\mathbb{R}^{+}=[0,+\infty)$, opt stands for sup or inf, $Z$ and $Y$ are Banach spaces, $S \subseteq Z$ is the state space, $D \subseteq Y$ is the decision space, $B(S)$ denotes the Banach space of all bounded real-valued functions on $S$ with norm

$$
\|w\|=\sup \{|w(x)|: x \in S\} \quad \text { for any } w \in B(S)
$$

and $u, v: S \times D \rightarrow \mathbb{R} ; a_{i}: S \times D \rightarrow S ; H_{i}: S \times D \times \mathbb{R} \rightarrow \mathbb{R}$. Also,

$$
\begin{gathered}
\Phi=\left\{\varphi: \varphi: \mathbb{R}^{+} \rightarrow \mathbb{R}^{+}\right. \text {is Lebesgue integrable with finite integral such that } \\
\left.\int_{0}^{\varepsilon} \varphi(t) d t>0 \text {, for each } \varepsilon>0\right\}
\end{gathered}
$$

and

$$
\begin{aligned}
& \Psi=\left\{\psi: \psi: \mathbb{R}^{+} \rightarrow \mathbb{R}^{+} \text {is upper semi-continuous on } \mathbb{R}^{+} \backslash\{0\}, \psi(0)=0\right. \\
& \quad \text { and } \psi(t)<t \text {, for each } t>0\} .
\end{aligned}
$$

(c) 2015 Sarwar et al. This article is distributed under the terms of the Creative Commons Attribution 4.0 International License (http://creativecommons.org/licenses/by/4.0/), which permits unrestricted use, distribution, and reproduction in any medium, provided you give appropriate credit to the original author(s) and the source, provide a link to the Creative Commons license, and indicate if changes were made. 
Fixed point theory is one of the most fruitful and applicable topics of nonlinear analysis, which is widely used not only in other mathematical theories, but also in many practical problems of natural sciences and engineering. The Banach contraction mapping principle [1] is indeed the most popular result of metric fixed point theory. This principle has many application in several domains, such as differential equations, functional equations, integral equations, economics, wild life, and several others.

Branciari [2] gave an integral version of the Banach contraction principles and proved fixed point theorem for a single-valued contractive mapping of integral type in metric space. Afterwards many researchers [3-18] extended the result of Branciari and obtained fixed point and common fixed point theorems for various contractive conditions of integral type on different spaces. In particular, Liu et al. [9] studied fixed point theorems satisfying a contractive condition of integral type and applied their results for the existence and uniqueness of a solution to the following functional equation:

$$
f(x)=\operatorname{opt}_{y \in D}\left\{u(x, y)+H_{1}\left(x, y, f_{1}\left(a_{1}(x, y)\right)\right)\right\} \quad \forall x \in Z .
$$

Further, Liu et al. [10] established common fixed point theorems satisfying contractive condition of integral type and applied their results for the existence and uniqueness of common solution to the following system of functional equations:

$$
\left\{\begin{array}{ll}
f(x)=\text { opt }_{y \in D}\left\{u(x, y)+H_{1}\left(x, y, f_{1}\left(a_{1}(x, y)\right)\right)\right\} & \forall x \in S, \\
g(x)=\text { opt }_{y \in D}\left\{v(x, y)+H_{2}\left(x, y, f_{2}\left(a_{2}(x, y)\right)\right)\right\} & \forall x \in S,
\end{array}\right\}
$$

where $x$ and $y$ signify the state and decision vectors, respectively, $a_{1}$ and $a_{2}$ represent the transformations of the process, $f_{1}(x)$ and $f_{2}(x)$ denote the optimal return functions with the initial state $x$.

The aim of this contribution is to study the existence and uniqueness of common solution for the system of functional equations arising in dynamic programming with the help of common fixed point results satisfying the contractive conditions of integral type in metric space.

Now, we recollect some known definitions and results from the literature which are helpful in the proof of our main results.

Definition 1.1 A coincidence point of a pair of self-mapping $K, L: X \rightarrow X$ is a point $x \in X$ for which $K x=L x$.

A common fixed point of a pair of self-mapping $K, L: X \rightarrow X$ is a point $x \in X$ for which $K x=L x=x$.

Jungck [19] initiated the concept of weakly compatible maps to study common fixed point theorems.

Definition 1.2 [19] A pair of self-mapping $K, L: X \rightarrow X$ is weakly compatible if they commute at their coincidence points, that is, if there exists a point $x \in X$ such that $K L x=L K x$ whenever $K x=L x$.

In the study of common fixed points of weakly compatible mappings, we often require the assumption of completeness of the space or subspace or continuity of mappings in- 
volved besides some contractive condition. Aamri and El Moutawakil [20] introduced the notion of (E.A) property, which requires only the closedness of the subspace and Liu et al. [21] extended the (E.A) property to common the (E.A) property as follows.

Definition 1.3 Let $(X, d)$ be a metric space and $K, L, M, N: X \rightarrow X$ be four self-maps. The pairs $(K, M)$ and $(L, N)$ satisfy the common (E.A) property if there exist two sequences $\left\{x_{n}\right\}$ and $\left\{y_{n}\right\}$ in $X$ such that

$$
\lim _{n \rightarrow \infty} K x_{n}=\lim _{n \rightarrow \infty} M x_{n}=\lim _{n \rightarrow \infty} L y_{n}=\lim _{n \rightarrow \infty} N y_{n}=t \in X .
$$

Sintunavarat and Kumam [22] introduced the notion of the (CLR) property, which never requires any condition on closedness of the space or subspace and Imdad et al. [23] introduced the common $(C L R)$ property which is an extension of the $(C L R)$ property.

Definition 1.4 Let $(X, d)$ be a metric space and $K, L, M, N: X \rightarrow X$ be four self maps. The pairs $(K, M)$ and $(L, N)$ satisfy the common limit range property with respect to mappings $M$ and $N$, denoted by $\left(C L R_{M N}\right)$ if there exist two sequences $\left\{x_{n}\right\}$ and $\left\{y_{n}\right\}$ in $X$ such that

$$
\lim _{n \rightarrow \infty} K x_{n}=\lim _{n \rightarrow \infty} M x_{n}=\lim _{n \rightarrow \infty} L y_{n}=\lim _{n \rightarrow \infty} N y_{n}=t \in M(X) \cap N(X) .
$$

Finally, we will need the following results.

Lemma 1.1 [9] Let $\varphi \in \Phi$ and $\left\{r_{n}\right\}_{n \in N}$ be a non-negative sequence with $\lim _{n \rightarrow \infty} r_{n}=a$. Then

$$
\lim _{n \rightarrow \infty} \int_{0}^{r_{n}} \varphi(t) d t=\int_{0}^{a} \varphi(t) d t
$$

Lemma 1.2 [24] Let $E$ be a set and $p, q: E \rightarrow \mathbb{R}$ be mappings. If opt $_{y \in E} p(y)$ and $\operatorname{opt}_{y \in E} q(y)$ are bounded, then

$$
\left|\mathrm{opt}_{y \in E} p(y)-\mathrm{opt}_{y \in E} q(y)\right| \leq \sup _{y \in E}|p(y)-q(y)| .
$$

\section{Common fixed point theorems}

In this section, we study common fixed point theorems for weakly compatible mappings using the common $(C L R)$ and common (E.A.) properties.

Theorem 2.1 Let $(X, d)$ be a metric space and $K, L, N, M: X \rightarrow X$ be four self-mappings satisfying the following conditions:

(1) the pairs $(K, N)$ and $(L, M)$ share $\left(C L R_{N M}\right)$ property;

(2)

$$
\int_{0}^{d(K x, L y)} \varphi(t) d t \leq \psi\left(\int_{0}^{\Delta_{1}(x, y)} \varphi(t) d t\right), \quad \forall x, y \in X
$$

where $(\varphi, \psi) \in \Phi \times \Psi$ and 


$$
\begin{aligned}
\triangle_{1}(x, y)=\max \{ & d(N x, M y), d(N x, K x), d(M y, L y), \frac{1}{2}[d(K x, M y)+d(L y, N x)], \\
& \frac{d(K x, N x) d(L y, M y)}{1+d(N x, M y)}, \frac{d(K x, M y) d(L y, N x)}{1+d(N x, M y)}, \\
& \left.d(N x, K x) \frac{1+d(N x, L y)+d(M y, K x)}{1+d(N x, K x)+d(M y, L y)}\right\} .
\end{aligned}
$$

If the pairs $(K, N)$ and $(L, M)$ are weakly compatible, then $K, L, M$, and $N$ have a unique common fixed point in $X$.

Proof Assume that the pairs $(K, N)$ and $(L, M)$ share the $\left(C L R_{N M}\right)$ property, then there exist two sequences $\left\{x_{n}\right\}$ and $\left\{y_{n}\right\}$ in $X$ such that

$$
\lim _{n \rightarrow \infty} K x_{n}=\lim _{n \rightarrow \infty} N x_{n}=\lim _{n \rightarrow \infty} L y_{n}=\lim _{n \rightarrow \infty} M y_{n}=z \quad \text { for some } z \in M(X) \cap N(X)
$$

Since $z \in N(X)$, there exists a point $u \in X$ such that $N u=z$. Thus (2.1) becomes

$$
\lim _{n \rightarrow \infty} K x_{n}=\lim _{n \rightarrow \infty} N x_{n}=\lim _{n \rightarrow \infty} L y_{n}=\lim _{n \rightarrow \infty} M y_{n}=z=N u
$$

Now, we claim that $K u=N u$. To prove the claim, let $K u \neq N u$. Then on putting $x=u$ and $y=y_{n}$ in condition (2) of Theorem 2.1, we have

$$
\int_{0}^{d\left(K u, L y_{n}\right)} \varphi(t) d t \leq \psi\left(\int_{0}^{\triangle_{1}\left(u, y_{n}\right)} \varphi(t) d t\right)
$$

where

$$
\begin{aligned}
\triangle_{1}\left(u, y_{n}\right)=\max \{ & d\left(N u, M y_{n}\right), d(N u, K u), d\left(M y_{n}, L y_{n}\right), \\
& \frac{1}{2}\left[d\left(K u, M y_{n}\right)+d\left(L y_{n}, N u\right)\right], \frac{d(K u, N u) d\left(L y_{n}, M y_{n}\right)}{1+d\left(N u, M y_{n}\right)}, \\
& \left.\frac{d\left(K u, M y_{n}\right) d\left(L y_{n}, N u\right)}{1+d\left(N u, M y_{n}\right)}, d(N u, K u) \frac{1+d\left(N u, L y_{n}\right)+d\left(M y_{n}, K u\right)}{1+d(N u, K u)+d\left(M y_{n}, L y_{n}\right)}\right\} .
\end{aligned}
$$

Taking the upper limit as $n \rightarrow \infty$ in equations (2.4) and (2.3), respectively, we have

$$
\lim _{n \rightarrow \infty} \triangle_{1}\left(u, y_{n}\right)=\max \left\{0, d(z, K u), 0, \frac{1}{2}[d(K u, z)], 0,0, d(z, K u)\right\}=d(K u, z)
$$

and

$$
\begin{aligned}
\int_{0}^{d(K u, z)} \varphi(t) d t & =\limsup _{n \rightarrow \infty} \int_{0}^{d\left(K u, L y_{n}\right)} \varphi(t) d t \\
& \leq \limsup _{n \rightarrow \infty} \psi\left(\int_{0}^{\triangle_{1}\left(u, y_{n}\right)} \varphi(t) d t\right) \leq \psi\left(\limsup _{n \rightarrow \infty} \int_{0}^{\triangle_{1}\left(u, y_{n}\right)} \varphi(t) d t\right) \\
& =\psi\left(\int_{0}^{d(K u, z)} \varphi(t) d t\right) \\
& <\int_{0}^{d(K u, z)} \varphi(t) d t
\end{aligned}
$$


which is a contradiction, thus $K u=N u$ and hence

$$
K u=N u=z .
$$

Similarly, since $z \in M(X)$, so there exists a point $v \in X$ such that $M v=z$. Thus (2.1) becomes

$$
\lim _{n \rightarrow \infty} K x_{n}=\lim _{n \rightarrow \infty} N x_{n}=\lim _{n \rightarrow \infty} L y_{n}=\lim _{n \rightarrow \infty} M y_{n}=z=M v
$$

Now, we claim that $L v=M v$. To support the claim, let $L v \neq M v$. Then on putting $x=x_{n}$ and $y=v$ in condition (2) of Theorem 2.1, one can get

$$
L v=M v=z
$$

Therefore, from (2.5) and (2.7), one can write

$$
K u=N u=L v=M v=z .
$$

Next, we show that $z$ is a common fixed point of $K, L, M$, and $N$. To this aim, since the pairs $(K, N)$ and $(L, M)$ are weakly compatible, then using (2.8) we have

$$
K u=N u \Rightarrow N K u=K N u \Rightarrow K z=N z \text {, }
$$

and

$$
L v=M v \quad \Rightarrow \quad M L v=L M v \quad \Rightarrow \quad L z=M z \text {. }
$$

We will show next that $K z=z$. Otherwise, if $K z \neq z$, using condition (2) of Theorem 2.1 with $x=z$ and $y=v$, we have

$$
\int_{0}^{d(K z, L v)} \varphi(t) d t \leq \psi\left(\int_{0}^{\triangle_{1}(z, v)} \varphi(t) d t\right)
$$

where

$$
\begin{aligned}
\triangle_{1}(z, v)=\max \{ & d(N z, M v), d(N z, K z), d(M v, L v), \\
& \frac{1}{2}[d(K z, M v)+d(L v, N z)], \frac{d(K z, N z) d(L v, M v)}{1+d(N z, M v)}, \\
& \left.\frac{d(K z, M v) d(L v, N z)}{1+d(N z, M v)}, d(N z, K z) \frac{1+d(N z, L v)+d(M v, K z)}{1+d(N z, K z)+d(M v, L v)}\right\} .
\end{aligned}
$$

In the light of (2.8) and (2.9), we get

$$
\begin{aligned}
\triangle_{1}(z, v) & =\max \left\{d(K z, z), 0,0, \frac{1}{2}[d(K z, z)+d(z, K z)], 0, \frac{d(K z, z) d(z, K z)}{1+d(K z, z)}, 0\right\} \\
& =d(K z, z)
\end{aligned}
$$


and

$$
\int_{0}^{d(K z, z)} \varphi(t) d t \leq \psi\left(\int_{0}^{d(K z, z)} \varphi(t) d t\right)<\int_{0}^{d(K z, z)} \varphi(t) d t
$$

which is a contradiction. Thus $K z=z$ and from (2.9), we can write

$$
K z=N z=z
$$

Similarly, setting $x=u, y=z$ in condition (2) of Theorem 2.1 and using (2.8), (2.10), one can get

$$
L z=M z=z \text {. }
$$

Therefore from (2.11) and (2.12), it follows that

$$
K z=L z=M z=N z=z
$$

that is, $z$ is a common fixed point of $K, L, M$, and $N$.

Finally, we prove the uniqueness of the common fixed point of $K, L, M$, and $N$. Assume that $z_{1}$ and $z_{2}$ are two distinct common fixed points of $K, L, M$, and $N$. Then replacing $x$ by $z_{1}$ and $y$ by $z_{2}$ in condition (2) of Theorem 2.1, we have

$$
\int_{0}^{d\left(z_{1}, z_{2}\right)} \varphi(t) d t=\int_{0}^{d\left(K z_{1}, L z_{2}\right)} \varphi(t) d t \leq \psi\left(\int_{0}^{\triangle_{1}\left(z_{1}, z_{2}\right)} \varphi(t) d t\right)
$$

where

$$
\begin{aligned}
\triangle_{1}\left(z_{1}, z_{2}\right)=\max \left\{d\left(N z_{1}, M z_{2}\right), d\left(N z_{1}, K z_{1}\right), d\left(M z_{2}, L z_{2}\right),\right. \\
\\
\qquad \frac{1}{2}\left[d\left(K z_{1}, M z_{2}\right)+d\left(L z_{2}, N z_{1}\right)\right], \frac{d\left(K z_{1}, N z_{1}\right) d\left(L z_{2}, M z_{2}\right)}{1+d\left(N z_{1}, M z_{2}\right)}, \\
\left.\qquad \frac{d\left(K z_{1}, M z_{2}\right) d\left(L z_{2}, N z_{1}\right)}{1+d\left(N z_{1}, M z_{2}\right)}, d\left(N z_{1}, K z_{1}\right) \frac{1+d\left(N z_{1}, L z_{2}\right)+d\left(M z_{2}, K z_{1}\right)}{1+d\left(N z_{1}, K z_{1}\right)+d\left(M z_{2}, L z_{2}\right)}\right\} \\
=\max \left\{d\left(z_{1}, z_{2}\right), 0,0, \frac{1}{2}\left[d\left(z_{1}, z_{2}\right)+d\left(z_{2}, z_{1}\right)\right], 0, \frac{d\left(z_{1}, z_{2}\right) d\left(z_{2}, z_{1}\right)}{1+d\left(z_{1}, z_{2}\right)}, 0\right\} \\
=d\left(z_{1}, z_{2}\right),
\end{aligned}
$$

so that

$$
\int_{0}^{d\left(z_{1}, z_{2}\right)} \varphi(t) d t \leq \psi\left(\int_{0}^{d\left(z_{1}, z_{2}\right)} \varphi(t) d t\right)<\int_{0}^{d\left(z_{1}, z_{2}\right)} \varphi(t) d t
$$

which is a contradiction and thus, $z_{1}=z_{2}$. Hence $K, L, M$, and $N$ have a unique common fixed point in $X$.

From Theorem 2.1, we easily deduce the following corollaries. 
Corollary 2.1 Let $(X, d)$ be a metric space and $K, N, M: X \rightarrow X$ be three self-mappings satisfying the following conditions:

(1) the pairs $(K, N)$ and $(K, M)$ share $\left(C K R_{N M}\right)$ property;

(2)

$$
\int_{0}^{d(K x, K y)} \varphi(t) d t \leq \psi\left(\int_{0}^{\Delta_{1}(x, y)} \varphi(t) d t\right), \quad \forall x, y \in X
$$

where $(\varphi, \psi) \in \Phi \times \Psi$ and

$$
\begin{gathered}
\triangle_{1}(x, y)=\max \left\{d(N x, M y), d(N x, K x), d(M y, K y), \frac{1}{2}[d(K x, M y)+d(K y, N x)],\right. \\
\frac{d(K x, N x) d(K y, M y)}{1+d(N x, M y)}, \frac{d(K x, M y) d(K y, N x)}{1+d(N x, M y)}, \\
\left.d(N x, K x) \frac{1+d(N x, K y)+d(M y, K x)}{1+d(N x, K x)+d(M y, K y)}\right\} .
\end{gathered}
$$

If the pairs $(K, N)$ and $(K, M)$ are weakly compatible, then $K, M$, and $N$ have a unique common fixed point in $X$.

Corollary 2.2 Let $(X, d)$ be a metric space and $K, M: X \rightarrow X$ be two self-mappings satisfying the following conditions:

(1) the pair $(K, M)$ satisfies the $\left(C L R_{M}\right)$ property;

(2)

$$
\int_{0}^{d(K x, K y)} \varphi(t) d t \leq \psi\left(\int_{0}^{\Delta_{1}(x, y)} \varphi(t) d t\right), \quad \forall x, y \in X
$$

where $(\varphi, \psi) \in \Phi \times \Psi$ and

$$
\begin{gathered}
\triangle_{1}(x, y)=\max \left\{d(M x, M y), d(M x, K x), d(M y, K y), \frac{1}{2}[d(K x, M y)+d(K y, M x)],\right. \\
\frac{d(K x, M x) d(K y, M y)}{1+d(M x, M y)}, \frac{d(K x, M y) d(K y, M x)}{1+d(M x, M y)}, \\
\left.d(M x, K x) \frac{1+d(M x, K y)+d(M y, K x)}{1+d(M x, K x)+d(M y, K y)}\right\} .
\end{gathered}
$$

If the pair $(K, M)$ is weakly compatible, then $K$ and $M$ have a unique common fixed point in $X$.

In a similar way to Theorem 2.1 the following result can be concluded and proved.

Theorem 2.2 Let $(X, d)$ be a metric space and $K, L, N, M: X \rightarrow X$ be four self-mappings satisfying the following conditions:

(1) the pairs $(K, N)$ and $(L, M)$ share $\left(C L R_{N M}\right)$ property;

(2)

$$
\int_{0}^{d(K x, L y)} \varphi(t) d t \leq \psi\left(\int_{0}^{\Delta_{2}(x, y)} \varphi(t) d t\right), \quad \forall x, y \in X
$$


where $(\varphi, \psi) \in \Phi \times \Psi$ and

$$
\begin{aligned}
& \triangle_{2}(x, y)=\max \left\{d(N x, M y), d(N x, K x), d(M y, L y), \frac{1}{2}[d(K x, M y)+d(L y, N x)],\right. \\
& \frac{d(K x, N x) d(L y, M y)}{1+d(K x, L y)}, \frac{d(K x, M y) d(L y, N x)}{1+d(K x, L y)}, \\
& \left.d(N x, K x) \frac{1+d(N x, L y)+d(M y, K x)}{1+d(N x, K x)+d(M y, L y)}\right\} .
\end{aligned}
$$

If the pairs $(K, N)$ and $(L, M)$ are weakly compatible, then $K, L, M$, and $N$ have a unique common fixed point in $X$.

Obviously, the $\left(C L R_{M N}\right)$ property implies the common property $(E . A)$ but the converse is not true in general. So replacing the $\left(C L R_{M N}\right)$ property by the common property $(E . A)$ in Theorem 2.1 and Theorem 2.2, we get the following results, the proofs of which can easily be done by following the lines of the proof of Theorem 2.1, because the (E.A) property together with the closedness property of a suitable subspace gives rise to the closed range property.

Corollary 2.3 Let $(X, d)$ be a metric space and $K, L, N, M: X \rightarrow X$ be four self-mappings satisfying the following conditions:

(1) the pairs $(K, N)$ and $(L, M)$ share common $(E . A)$ property such that $M(X)($ or $N(X))$ is closed subspace of $X$;

(2)

$$
\int_{0}^{d(K x, L y)} \varphi(t) d t \leq \psi\left(\int_{0}^{\Delta_{1}(x, y)} \varphi(t) d t\right), \quad \forall x, y \in X
$$

where $(\varphi, \psi) \in \Phi \times \Psi$ and

$$
\begin{aligned}
& \triangle_{1}(x, y)=\max \left\{d(N x, M y), d(N x, K x), d(M y, L y), \frac{1}{2}[d(K x, M y)+d(L y, N x)],\right. \\
& \frac{d(K x, N x) d(L y, M y)}{1+d(N x, M y)}, \frac{d(K x, M y) d(L y, N x)}{1+d(N x, M y)}, \\
& \left.d(N x, K x) \frac{1+d(N x, L y)+d(M y, K x)}{1+d(N x, K x)+d(M y, L y)}\right\} .
\end{aligned}
$$

If the pairs $(K, N)$ and $(L, M)$ are weakly compatible, then $K, L, M$, and $N$ have a unique common fixed point in $X$.

Corollary 2.4 Let $(X, d)$ be a metric space and $K, L, N, M: X \rightarrow X$ be four self-mappings satisfying the following conditions:

(1) the pairs $(K, N)$ and $(L, M)$ share common (E.A) property such that $M(X)($ or $N(X))$ is closed subspace of $X$;

(2)

$$
\int_{0}^{d(K x, L y)} \varphi(t) d t \leq \psi\left(\int_{0}^{\Delta_{2}(x, y)} \varphi(t) d t\right), \quad \forall x, y \in X
$$


where $(\varphi, \psi) \in \Phi \times \Psi$ and

$$
\begin{aligned}
& \triangle_{2}(x, y)=\max \left\{d(N x, M y), d(N x, K x), d(M y, L y), \frac{1}{2}[d(K x, M y)+d(L y, N x)],\right. \\
& \frac{d(K x, N x) d(L y, M y)}{1+d(K x, L y)}, \frac{d(K x, M y) d(L y, N x)}{1+d(K x, L y)}, \\
& \left.d(N x, K x) \frac{1+d(N x, L y)+d(M y, K x)}{1+d(N x, K x)+d(M y, L y)}\right\} .
\end{aligned}
$$

If the pairs $(K, N)$ and $(L, M)$ are weakly compatible, then $K, L, M$, and $N$ have a unique common fixed point in $X$.

One can obtain further consequences from Theorem 2.2 and Corollaries 2.3 and 2.4 in a similar way to Theorem 2.1.

Remark 2.1 Theorem 2.1 and Corollary 2.3 are still valid, if we replace $\triangle_{1}(x, y)$ by

$$
\begin{gathered}
\triangle_{3}(x, y)=\max \left\{d(N x, M y), d(N x, K x), d(M y, L y), \frac{1}{2}[d(K x, M y)+d(L y, N x)],\right. \\
\min \left(\frac{d(K x, N x) d(L y, M y)}{1+d(N x, M y)}, \frac{d(K x, M y) d(L y, N x)}{1+d(N x, M y)},\right. \\
\left.\left.d(N x, K x) \frac{1+d(N x, L y)+d(M y, K x)}{1+d(N x, K x)+d(M y, L y)}\right)\right\} .
\end{gathered}
$$

Similarly, Theorem 2.2 and Corollary 2.4 are still valid, if we replace $\triangle_{1}(x, y)$ by

$$
\begin{gathered}
\triangle_{4}(x, y)=\max \left\{d(N x, M y), d(N x, K x), d(M y, L y), \frac{1}{2}[d(K x, M y)+d(L y, N x)],\right. \\
\min \left(\frac{d(K x, N x) d(L y, M y)}{1+d(K x, L y)}, \frac{d(K x, M y) d(L y, N x)}{1+d(K x, L y)},\right. \\
\left.\left.d(N x, K x) \frac{1+d(N x, L y)+d(M y, K x)}{1+d(N x, K x)+d(M y, L y)}\right)\right\} .
\end{gathered}
$$

Finally, by choosing $K=L$ and $N$ and $M$ as identity mappings, we conclude some fixed point theorems for integral type contraction from our main Theorem 2.1, which can be listed as follows.

Corollary 2.5 Let $(X, d)$ be a metric space and $K: X \rightarrow X$ be a self-mapping satisfying the condition

$$
\int_{0}^{d(K x, K y)} \varphi(t) d t \leq \psi\left(\int_{0}^{\Delta_{1}(x, y)} \varphi(t) d t\right), \quad \forall x, y \in X
$$

where $(\varphi, \psi) \in \Phi \times \Psi$ and

$$
\begin{aligned}
\triangle_{1}(x, y)=\max \left\{d(x, y), d(x, K x), d(y, K y), \frac{1}{2}[d(K x, y)+d(K y, x)],\right. \\
\left.\quad \frac{d(K x, x) d(K y, y)}{1+d(x, y)}, \frac{d(K x, y) d(K y, x)}{1+d(x, y)}, d(x, K x) \frac{1+d(x, K y)+d(y, K x)}{1+d(x, K x)+d(y, K y)}\right\},
\end{aligned}
$$

for all $x, y \in X$. Then $K$ has a unique fixed point in $X$. 
Corollary 2.6 Let $(X, d)$ be a metric space and $K: X \rightarrow X$ be a self-mapping satisfying the condition

$$
\int_{0}^{d(K x, K y)} \varphi(t) d t \leq \psi\left(\int_{0}^{\Delta_{2}(x, y)} \varphi(t) d t\right), \quad \forall x, y \in X
$$

where $(\varphi, \psi) \in \Phi \times \Psi$ and

$$
\begin{aligned}
\triangle_{2}(x, y)=\max \left\{d(x, y), d(x, K x), d(y, K y), \frac{1}{2}[d(K x, y)+d(K y, x)],\right. \\
\left.\quad \frac{d(K x, x) d(K y, y)}{1+d(K x, y)}, \frac{d(K x, y) d(K y, x)}{1+d(K x, y)}, d(x, K x) \frac{1+d(x, K y)+d(y, K x)}{1+d(x, K x)+d(y, K y)}\right\},
\end{aligned}
$$

for all $x, y \in X$. Then $K$ has a unique fixed point in $X$.

Remark 2.2 Notice that several fixed point theorems such as the celebrated Banach fixed point theorem, fixed point theorems for Kannan, Chatterjee, and Reich type mappings and others can be deduced as particular cases of Corollary 2.5.

To illustrate Theorem 2.1, we construct the following example.

Example 2.1 Let $X=(0,2)$ be a metric space with metric $d(x, y)=|x-y|$, where $x, y \in X$ and $K, L, M, N$ be self-maps of $X$, defined by

$$
\begin{aligned}
& K x=\left\{\begin{array}{ll}
1 & \text { if } x \in(0,1], \\
\frac{1}{6} & \text { if } x \in(1,2)
\end{array} ; \quad L x= \begin{cases}1 & \text { if } x \in(0,1], \\
\frac{1}{8} & \text { if } x \in(1,2),\end{cases} \right. \\
& M x=\left\{\begin{array}{ll}
1 & \text { if } x \in(0,1], \\
\frac{1}{2} & \text { if } x \in(1,2),
\end{array} \text { and } \quad N x= \begin{cases}1 & \text { if } x \in(0,1], \\
\frac{1}{3} & \text { if } x \in(1,2) .\end{cases} \right.
\end{aligned}
$$

First we verify condition (1) of Theorem 2.1. To this aim, let $\left\{x_{n}\right\}=\left\{\frac{n}{n+1}\right\}_{n \geq 1}$ and $\left\{y_{n}\right\}=$ $\left\{\frac{1}{n+1}\right\}_{n \geq 1}$ be two sequences in $X$. Then

$$
\begin{aligned}
& \lim _{n \rightarrow \infty} K x_{n}=\lim _{n \rightarrow \infty} K\left(\frac{n}{n+1}\right)=1 ; \\
& \lim _{n \rightarrow \infty} L y_{n}=\lim _{n \rightarrow \infty} L\left(\frac{1}{n+1}\right)=1 ; \\
& \lim _{n \rightarrow \infty} M y_{n}=\lim _{n \rightarrow \infty} M\left(\frac{1}{n+1}\right)=1 ; \\
& \lim _{n \rightarrow \infty} N x_{n}=\lim _{n \rightarrow \infty} N\left(\frac{n}{n+1}\right)=1 .
\end{aligned}
$$

Thus

$$
\lim _{n \rightarrow \infty} K x_{n}=\lim _{n \rightarrow \infty} N x_{n}=\lim _{n \rightarrow \infty} L y_{n}=\lim _{n \rightarrow \infty} M y_{n}=1 \in M(X) \cap N(X) .
$$

That is, $(K, N)$ and $(K, M)$ satisfies the common $\left(C L R_{M N}\right)$ property. 
Next, to verify condition (2) of Theorem 2.1 let us define $\varphi: \mathbb{R}^{+} \rightarrow \mathbb{R}^{+}$by $\varphi(t)=2 t$ and $\psi: \mathbb{R}^{+} \rightarrow \mathbb{R}^{+}$by $\psi(t)=\frac{t}{5}$.

If $x, y \in(0,1]$. Then $K x=L y=M y=N x=1$ and

$$
\int_{0}^{d(K x, L y)} \varphi(t) d t=0=\psi\left(\int_{0}^{\Delta_{1}(x, y)} \varphi(t) d t\right)
$$

where $\Delta_{1}(x, y)=0$.

$$
\begin{aligned}
& \text { If } x, y \in(1,2) \text {. Then } K x=\frac{1}{6}, L y=\frac{1}{8}, M y=\frac{1}{2}, N x=\frac{1}{3} \text {, and } \\
& \int_{0}^{d(K x, L y)} \varphi(t) d t=\int_{0}^{\frac{1}{24}} 2 t d t=\left.t^{2}\right|_{0} ^{\frac{1}{24}}=\frac{1}{576} .
\end{aligned}
$$

Also,

$$
\triangle_{1}(x, y)=\max \left\{\frac{1}{6}, \frac{1}{6}, \frac{3}{8}, \frac{13}{48}, \frac{3}{50}, \frac{1}{15}, \frac{1}{6}\right\}=\frac{3}{8} .
$$

Thus we obtain

$$
\psi\left(\int_{0}^{\Delta_{1}(x, y)} \varphi(t) d t\right)=\psi\left(\int_{0}^{\frac{3}{8}} 2 t d t\right)=\psi\left(\left.t^{2}\right|_{0} ^{\frac{3}{8}}\right)=\frac{9}{320}>\int_{0}^{d(K x, L y)} \varphi(t) d t
$$

Hence from the above two cases it follows that

$$
\int_{0}^{d(K x, L y)} \varphi(t) d t \leq \psi\left(\int_{0}^{\Delta_{1}(x, y)} \varphi(t) d t\right), \quad \forall x, y \in X
$$

Therefore from Theorem 2.1, $K, L, M$, and $N$ have a unique common fixed point, which is $x=1$.

\section{Applications to existence theorems for functional equations arising in dynamic programming}

In this section, an attempt is made to find the existence and uniqueness of a common solution for a system of functional equations arising in dynamic programming through the help of Theorem 2.1. Consider the system

$$
\left\{\begin{array}{ll}
f_{1}(x)=\text { opt }_{y \in D}\left\{u(x, y)+H_{1}\left(x, y, f_{1}\left(a_{1}(x, y)\right)\right)\right\} & \forall x \in S, \\
f_{2}(x)=\text { opt }_{y \in D}\left\{u(x, y)+H_{2}\left(x, y, f_{2}\left(a_{2}(x, y)\right)\right)\right\} & \forall x \in S, \\
f_{3}(x)=\text { opt }_{y \in D}\left\{v(x, y)+H_{3}\left(x, y, f_{3}\left(a_{3}(x, y)\right)\right)\right\} & \forall x \in S, \\
f_{4}(x)=\text { opt }_{y \in D}\left\{v(x, y)+H_{4}\left(x, y, f_{4}\left(a_{4}(x, y)\right)\right)\right\} & \forall x \in S,
\end{array}\right\}
$$

where $x$ and $y$ signify the state and decision vectors, respectively, $a_{1}, a_{2}, a_{3}$, and $a_{4}$ represent the transformations of the process, $f_{1}(x), f_{2}(x), f_{3}(x)$, and $f_{4}(x)$ denote the optimal return functions with the initial state $x$. 
Let $K, L, M, N: B(S) \rightarrow B(S)$ be the mappings defined by

$$
\begin{aligned}
& K h(x)=\operatorname{opt}_{y \in D}\left\{u(x, y)+H_{1}\left(x, y, h\left(a_{1}(x, y)\right)\right)\right\}, \\
& L h(x)=\operatorname{opt}_{y \in D}\left\{u(x, y)+H_{2}\left(x, y, h\left(a_{2}(x, y)\right)\right)\right\}, \\
& M h(x)=\operatorname{opt}_{y \in D}\left\{v(x, y)+H_{3}\left(x, y, h\left(a_{3}(x, y)\right)\right)\right\}, \\
& N h(x)=\operatorname{opt}_{y \in D}\left\{v(x, y)+H_{4}\left(x, y, h\left(a_{4}(x, y)\right)\right)\right\},
\end{aligned}
$$

where $(x, h) \in S \times B(S)$.

Theorem 3.1 Let $K, L, M, N: B(S) \rightarrow B(S)$ given by (3.2) be mappings for which the following conditions hold:

(1) $u, v$, and $H_{i}$ are bounded for $i=1,2,3,4$;

(2) the pairs $(K, N)$ and $(L, M)$ share $\left(C L R_{N M}\right)$ property;

(3) for some $h \in B(S), K N h=N K h$, whenever $K h=N h$ and $L M h=M L h$, whenever $L h=M h$;

(4) for all $(x, y, h, w) \in S \times D \times B(S) \times B(S)$,

$$
\int_{0}^{\left|H_{1}\left(x, y, h\left(a_{1}(x, y)\right)\right)-H_{2}\left(x, y, w\left(a_{2}(x, y)\right)\right)\right|} \varphi(t) d t \leq \psi\left(\int_{0}^{\Delta_{1}^{*}(h, w)} \varphi(t) d t\right),
$$

where

$$
\begin{aligned}
\Delta_{1}^{*}(h, w)=\max \{ & \|N h-M w\|,\|N h-K h\|,\|M w-L w\|, \\
& \frac{1}{2}[\|K h-M w\|+\|L w-N h\|], \frac{\|K h-N h\|\|L w-M w\|}{1+\|N h-M w\|}, \\
& \frac{\|K h-M w\|\|L w-N h\|}{1+\|N h-M w\|}, \\
& \left.\|N h-K h\| \frac{1+\|N h-L w\|+\|M w-K h\|}{1+\|N h-K h\|+\|M w-L w\|}\right\} .
\end{aligned}
$$

Then the system of functional equations (3.1) has a unique common solution in $B(S)$.

Proof Since $u, v$, and $H_{i}$ are bounded for $i=1,2,3,4$, there exists $M>0$ such that

$$
\sup \left\{\|u(x, y)\|,\|v(x, y)\|,\left\|H_{i}(x, y, t)\right\|:(x, y, t) \in S \times D \times R\right\} \leq M
$$

Thus by (3.2), (3.3), and Lemma 1.2, $K, L, M, N$ are self-mappings in $B(S)$.

Let $(x, h, w) \in S \times B(S) \times B(S)$. Suppose that opt $\mathrm{y}_{y \in D}=\inf _{y \in D}$. Then using (3.2) we can find $y, z \in D$ such that

$$
\begin{aligned}
& K h(x)>u(x, y)+H_{1}\left(x, y, h\left(a_{1}(x, y)\right)\right)-\delta ; \\
& L w(x)>u(x, z)+H_{2}\left(x, z, w\left(a_{2}(x, z)\right)\right)-\delta ; \\
& K h(x) \leq u(x, z)+H_{1}\left(x, z, h\left(a_{1}(x, z)\right)\right) ; \\
& L w(x) \leq u(x, y)+H_{2}\left(x, y, w\left(a_{2}(x, y)\right)\right) ;
\end{aligned}
$$

where $(x, h) \in S \times B(S)$. 
Next, with the help of (3.4) and (3.7), we have

$$
\begin{aligned}
& K h(x)-L w(x)> H_{1}\left(x, y, h\left(a_{1}(x, y)\right)\right)-H_{2}\left(x, y, w\left(a_{2}(x, y)\right)\right)-\delta \\
& \geq-\max \left\{\left|H_{1}\left(x, y, h\left(a_{1}(x, y)\right)\right)-H_{2}\left(x, y, w\left(a_{2}(x, y)\right)\right)\right|,\right. \\
&\left.\left|H_{1}\left(x, z, h\left(a_{1}(x, z)\right)\right)-H_{2}\left(x, z, w\left(a_{2}(x, z)\right)\right)\right|\right\}-\delta .
\end{aligned}
$$

Analogously, with the help of (3.5) and (3.6), we have

$$
\begin{aligned}
K h(x)-L w(x)< & H_{1}\left(x, z, h\left(a_{1}(x, z)\right)\right)-H_{2}\left(x, z, w\left(a_{2}(x, z)\right)\right)+\delta \\
\leq & \max \left\{\left|H_{1}\left(x, y, h\left(a_{1}(x, y)\right)\right)-H_{2}\left(x, y, w\left(a_{2}(x, y)\right)\right)\right|\right. \\
& \left.\left|H_{1}\left(x, z, h\left(a_{1}(x, z)\right)\right)-H_{2}\left(x, z, w\left(a_{2}(x, z)\right)\right)\right|\right\}+\delta
\end{aligned}
$$

So we can write

$$
\begin{gathered}
|K h(x)-L w(x)|<\max \left\{\left|H_{1}\left(x, y, h\left(a_{1}(x, y)\right)\right)-H_{2}\left(x, y, w\left(a_{2}(x, y)\right)\right)\right|,\right. \\
\left.\left|H_{1}\left(x, z, h\left(a_{1}(x, z)\right)\right)-H_{2}\left(x, z, w\left(a_{2}(x, z)\right)\right)\right|\right\}+\delta \\
=\max \left\{\left|H_{1}\left(x, y, h\left(a_{1}(x, y)\right)\right)-H_{2}\left(x, y, w\left(a_{2}(x, y)\right)\right)\right|+\delta,\right. \\
\left.\left|H_{1}\left(x, z, h\left(a_{1}(x, z)\right)\right)-H_{2}\left(x, z, w\left(a_{2}(x, z)\right)\right)\right|+\delta\right\}, \\
|K h(x)-L w(x)|<\max \{|A-B|+\delta,|C-D|+\delta\},
\end{gathered}
$$

where $A=H_{1}\left(x, y, h\left(a_{1}(x, y)\right)\right), B=H_{2}\left(x, y, w\left(a_{2}(x, y)\right)\right), C=H_{1}\left(x, z, h\left(a_{1}(x, z)\right)\right)$, and $D=$ $H_{2}\left(x, z, w\left(a_{2}(x, z)\right)\right)$.

Similarly, one can obtain (3.8), if opt $\mathrm{y \in D}=\sup _{y \in D}$. Now, using (3.8), we have

$$
\begin{aligned}
\int_{0}^{|K h(x)-L w(x)|} \varphi(t) d t \leq & \int_{0}^{\max \{|A-B|+\delta,|C-D|+\delta\}} \varphi(t) d t \\
& =\max \left\{\int_{0}^{|A-B|+\delta} \varphi(t) d t, \int_{0}^{|C-D|+\delta} \varphi(t) d t\right\} \\
& =\max \left\{\int_{0}^{|A-B|} \varphi(t) d t+\int_{|A-B|}^{|A-B|+\delta} \varphi(t) d t\right. \\
= & \max \left\{\int_{0}^{|A-B|} \varphi(t) d t, \int_{0}^{|C-D|} \varphi(t) d t\right\} \\
& +\max \left\{\int_{|A-B|}^{|C-D|} \varphi(t) d t+\int_{|C-D|}^{|C-D|+\delta} \varphi(t) d t\right\}
\end{aligned}
$$

and by condition (4) of Theorem 3.1, we get

$$
\int_{0}^{\|K h-L w\|} \varphi(t) d t \leq \psi\left(\int_{0}^{\triangle_{1}^{*}(h, w)} \varphi(t) d t\right)+\max \left\{\int_{|A-B|}^{|A-B|+\delta} \varphi(t) d t, \int_{|C-D|}^{|C-D|+\delta} \varphi(t) d t\right\}
$$

where $(x, h, w) \in S \times B(S) \times B(S)$. 
In the light of (3.3), Theorem 12.34 in [25] and $\varphi \in \Phi$, for each $\varepsilon>0$, we can find $\delta \in$ $(0, M)$ satisfying

$$
\int_{C} \varphi(t) d t \leq \varepsilon, \quad \forall C \subseteq[0,3 M] \text { with } m(C) \leq \delta,
$$

where $m(C)$ denotes the Lebesgue measure of $C$. Thus (3.9) becomes

$$
\int_{0}^{\|K h-L w\|} \varphi(t) d t \leq \psi\left(\int_{0}^{\triangle_{1}^{*}(h, w)} \varphi(t) d t\right)+\varepsilon, \quad \forall h, w \in B(S) .
$$

Taking the limit as $\varepsilon \rightarrow 0^{+}$, we get

$$
\int_{0}^{\|K h-L w\|} \varphi(t) d t \leq \psi\left(\int_{0}^{\triangle_{1}^{*}(h, w)} \varphi(t) d t\right), \quad \forall h, w \in B(S) .
$$

Thus all the conditions of Theorem 2.1 are satisfied. Hence the mappings $K, L, M, N$ have a unique common fixed point in $B(S)$, that is, the system of functional equations (3.1) has a unique common solution.

\section{Competing interests}

The authors declare that they have no competing interests regarding this manuscript.

\section{Authors' contributions}

All authors read and approved the final version.

\section{Author details}

'Department of Mathematics, University of Malakand, Chakdara Dir(L), Pakistan. ${ }^{2}$ Department of Mathematics, Atılım University, Ankara, Turkey.

\section{Acknowledgements}

The authors are grateful to the editor and anonymous reviewers for their careful reviews, valuable comments, and remarks to improve this paper.

\section{Received: 16 September 2015 Accepted: 10 November 2015 Published online: 25 November 2015}

\section{References}

1. Banach, S: Sur les opérations dans les ensembles abstraits et leurs applications aux equations integrales. Fundam. Math. 3, 133-181 (1922)

2. Branciari, A: A fixed point theorem for mappings satisfying a general contractive condition of integral type. Int. J. Math. Math. Sci. 29(9), 531-536 (2002)

3. Aliouche, A: A common fixed point theorem for weakly compatible mappings in symmetric spaces satisfying a contractive condition of integral type. J. Math. Anal. Appl. 322, 796-802 (2006)

4. Altun, I, Türkoglu, D: Some fixed point theorems for weakly compatible mappings satisfying an implicit relation. Taiwan. J. Math. 13, 1291-1304 (2009)

5. Altun, I, Türkoglu, D, Rhoades, BE: Fixed points of weakly compatible maps satisfying a general contractive of integral type. Fixed Point Theory Appl. 2007, Article ID 17301 (2007)

6. Djoudi, A, Aliouche, A: Common fixed point theorems of Greguš type for weakly compatible mappings satisfying contractive conditions of integral type. J. Math. Anal. Appl. 329, 31-45 (2007)

7. Djoudi, A, Merghadi, F: Common fixed point theorems for maps under a contractive condition of integral type. J. Math. Anal. Appl. 341, 953-960 (2008)

8. Jachymski, J: Remarks on contractive conditions of integral type. Nonlinear Anal. 71, 1073-1081 (2009)

9. Liu, Z, Li, X, Kang, SM, Cho, SY: Fixed point theorems for mappings satisfying contractive conditions of integral type and applications. Fixed Point Theory Appl. 2001, Article ID 64 (2011)

10. Liu, Z, Zou, X, Kang, SM, Ume, JS: Common fixed points for a pair of mappings satisfying contractive conditions of integral type. J. Inequal. Appl. 2014, Article ID 394 (2014)

11. Murthy, PP, Kumar, S, Tas, K: Common fixed points of self maps satisfying an integral type contractive condition in fuzzy metric spaces. Math. Commun. 15, 521-537 (2010)

12. Sintunavarat, $W$, Kumam, P: Gregus-type common fixed point theorems for tangential multi-valued mappings of integral type in metric spaces. Int. J. Math. Math. Sci. 2011, Article ID 923458 (2011)

13. Alsulami, HH, Karapınar, E, O'Regan, D, Shahi, P: Fixed points of generalized contractive mappings of integral type. Fixed Point Theory Appl. 2014, Article ID 213 (2014) 
14. Karapınar, E, Shahi, P, Tas, K: Generalized $\alpha$ - $\psi$-contractive type mappings of integral type and related fixed point theorems. J. Inequal. Appl. 2014, Article ID 160 (2014)

15. Chauhan, S, Karapınar, E: Some integral type common fixed point theorems satisfying $\Psi$-contractive conditions. Bull. Belg. Math. Soc. Simon Stevin 21(4), 593-612 (2014)

16. Gulyaz, S, Karapınar, E, Rakocevic, V, Salimi, P: Existence of a solution of integral equations via fixed point theorem. J. Inequal. Appl. 2013, Article ID 529 (2013)

17. Karapınar, E: Fixed points results for alpha-admissible mapping of integral type on generalized metric spaces. Abstr. Appl. Anal. 2014, Article ID 141409 (2014)

18. Chauhan, S, Imdad, M, Karapınar, E, Fisher, B: An integral type fixed point theorem for multi-valued mappings employing strongly tangential property. J. Egypt. Math. Soc. 22(2), 258-264 (2014)

19. Jungck, G: Common fixed points for non-continuous non-self mappings on a non-numeric spaces. Far East J. Math. Sci. 4(2), 199-212 (1996)

20. Aamri, M, El Moutawakil, D: Some new common fixed point theorems under strict contractive conditions. J. Math Anal. Appl. 270(1), 181-188 (2002)

21. Liu, W, Wu, J, Li, Z: Common fixed points of single-valued and multi-valued maps. Int. J. Math. Math. Sci. 19, 3045-3055 (2005)

22. Sintunavarat, W, Kumam, P: Common fixed point theorem for a pair of weakly compatible mappings in fuzzy metric space. J. Appl. Math. 2011, Article ID 637958 (2011)

23. Imdad, $\mathrm{M}$, Pant, $\mathrm{BD}$, Chauhan, S: Fixed point theorems in Menger spaces using the (CLR $\left.R_{S T}\right)$ property and applications. J. Nonlinear Anal. Optim. 3(2), 225-237 (2012)

24. Liu, Z, Kang, SM: Existence and uniqueness of solutions for two classes of functional equations arising in dynamic programming. Acta Math. Appl. Sinica (Engl. Ser.) 23, 195-208 (2007)

25. Hewitt, K, Stromberg, K: Real and Abstract Analysis. Springer, New York (1978)

\section{Submit your manuscript to a SpringerOpen ${ }^{\odot}$ journal and benefit from:}

- Convenient online submission

- Rigorous peer review

- Immediate publication on acceptance

- Open access: articles freely available online

- High visibility within the field

- Retaining the copyright to your article 